\title{
Joint Topology Control and Routing in IEEE 802.11-Based Multiradio Multichannel Mesh Networks
}

\author{
Lin Chen, Qian Zhang, Senior Member, IEEE, Minglu Li, Associate Member, IEEE, and Weijia Jia
}

\begin{abstract}
Due to low cost, ease of deployment, increased coverage, and enhanced capacity, multiradio mesh networks that utilize inexpensive and readily available Institute of Electrical and Electronics Engineers (IEEE) 802.11 wireless interfaces are touted as the new frontier of wireless networking. In a multihop mesh system, the close interaction between topology control and routing selection affects the system throughput of a wireless network. This paper proposes a novel joint topology control and routing (JTCR) protocol for a multiradio multichannel wireless mesh network to exploit both channel diversity and spatial reusability. It resides between medium access control and the network layer and aims to improve the network throughput by coordinating transmission power, channel assignment, and route selection among multiple nodes in a distributed way. JTCR jointly coordinates the transmission power at each node, the channel selection on each wireless interface, and the route selection among interfaces based on the traffic information that is measured and exchanged among two-hop neighbor nodes. An equivalent channel air time metric $(E C A T M)$ is presented to quantify the difference of various adjustment candidates. This protocol achieves the efficient utilization of available channels by selecting a feasible adjustment candidate with the smallest $E C A T M$ value and coordinating affected nodes to realize the adjustment. Our NS-2-based simulation results show that the network throughput can be significantly improved by using our proposed solution.
\end{abstract}

Index Terms-Distributed algorithm, IEEE 802.11, routing, topology control, wireless mesh.

\section{INTRODUCTION}

W IRELESS mesh networks are built on a mix of fixed and mobile nodes that are interconnected via wireless links to form a multihop network [1]. These networks have attracted increasing attracted increasing research interest due to

Manuscript received April 27, 2006; revised September 12, 2006 and January 9, 2007. This work was supported in part by the National Basic Research Program of China under 973 Program 2006CB303000, the National 863 Program of China under Grant 2006AA01Z228, the Key Project of Guangzhou Municipal Government Guangdong/Hong Kong Critical Technology under Grant 2006Z1-D6131, and the National Natural Science Foundation of China under Grants 60473092 and 90612018 . The review of this paper was coordinated by Prof. X. Zhang.

L. Chen and M. Li are with the Department of Computer Science and Engineering, Shanghai Jiao Tong University, Shanghai 200240, China (e-mail: chen.lin@sjtu.edu.cn; li-ml@cs.sjtu.edu.cn).

Q. Zhang is with the Department of Computer Science, Hong Kong University of Science and Technology, Kowloon, Hong Kong (e-mail: qianzh@ cs.ust.hk).

W. Jia is with the Department of Computer Science, City University of Hong Kong, Kowloon, Hong Kong (e-mail: wjia@cs.cityu.edu.hk).

Color versions of one or more of the figures in this paper are available online at http://ieeexplore.ieee.org.

Digital Object Identifier 10.1109/TVT.2007.900509 their potential applications, which include last-mile broadband Internet access, community sharing, neighborhood gaming, and so on. Due to the broadcast nature of a wireless medium, the limited channel capacity, and the influence of cochannel interference, improving network throughput becomes a critical requirement in such networks.

According to the Institute of Electrical and Electronics Engineers (IEEE), standard 802.11 offers multiple orthogonal channels that can simultaneously work within a neighborhood [2], [3]. Meanwhile, the price for commodity 802.11 radios is rapidly diminishing. These make it natural to consider the use of multiple inexpensive radios at a single node, where each radio is designed to be capable of switching over multiple orthogonal channels. By allowing multiple simultaneous transmissions within a neighborhood, the cochannel interference is alleviated. Consequently, it is expected that higher system throughput can be achieved.

A previous work in this area mainly focused on exploiting the channel diversity characteristic to improve system performance. Channel assignment algorithms [4]-[6], routing protocols [7], [8], or joint channel assignment and routing algorithms [9]-[11] for multiradio multichannel multihop networks have been explored. Due to the limited number of available channels, cochannel interference can only be reduced to a certain degree. In order to further improve the network throughput, we need to seek other way out. Another way to mitigate cochannel interference is to leverage spatial reusability. The idea is to adjust the power level of each radio according to the current channel conditions to increase the opportunity of channel spatial reusability. In this paper, we focus on considering both channel diversity and spatial reusability to reduce cochannel interference by jointly adjusting channel, transmission power, and routing. Since both channel assignment and power control affect network topology, we jointly regard them as a topology control problem.

In wireless mesh networks, topology control, which further consists of channel assignment as well as power control, and routing are coupled together. On one hand, channel assignment and power control determine the connectivity between nodes since two nodes can communicate with each other only when they are on a common channel and within the transmission range of each other. As we know, routing decisions are made based on the network topology. Thus, channel assignment and power control have a direct impact on routing. On the other hand, to achieve a better result, channel and transmission power should be dynamically adjusted according to the traffic status, 
which is determined by a routing algorithm. Therefore, routing, channel assignment, and power control are tightly coupled and should be jointly optimized.

To our best knowledge, this is the first paper that has addressed joint topology control and the routing (JTCR) problem in an IEEE 802.11-based multiradio multichannel wireless mesh network. We propose and evaluate a novel JTCR protocol, which resides between medium-access control (MAC) and network layer, to coordinate transmission power, channel assignment, and route selection among multiple nodes. Since we may have various adjustment candidates that represent specified combined solutions of topology and/or route change, a uniformly quantified metric is needed for a node to evaluate the channel condition and to quantify the potential performance gain of different candidates. In this paper, we first present a novel equivalent channel air time metric $(E C A T M)$ that takes into account not only link bandwidth, packet loss probability, and channel utilization but also channel spatial reusability. This metric is utilized to evaluate the total air time consumption for a specific adjustment candidate. Smaller value represents less cochannel interference. Based on the performance evaluation metric and adjustment candidates, we propose a distributed algorithm running on every node, which periodically checks the channel utilization condition. When channel overloaded condition is detected, this algorithm tries to find the best adjustment candidate in terms of the smallest ECATM metric value and then makes the corresponding negotiation and adjustment. An adjustment candidate should at least observe the following three constraints before we call it a feasible candidate. First, the new candidate should maintain the current throughput achieved by each flow. Then, considering the potential risk of network partition, the adjustment candidate should preserve network connectivity. Finally, the adjustment of power levels should not cause asymmetric links, which exacerbate the hidden terminal problem. Through an extensive simulation study, we show that the JTCR algorithm can significantly improve aggregated throughput with low overhead.

The rest of this paper is organized as follows. The motivating scenario for joint topology and routing is described in Section II. Section III states the system model and assumptions. The rationale for the performance metric design is discussed in Section IV. We present the distributed JTCR algorithm in Section V. Section VI describes the system implementation. Simulation results are presented in Section VII. A related work is addressed in Section VIII. Finally, Section IX closes this paper with some concluding remarks and future research directions.

\section{Motivating SCEnARIO}

To illustrate the significance of JTCR, it is helpful to have a real-world scenario in mind. Consider a network topology composed of ten wireless nodes $\left(N_{1}-N_{10}\right)$, as shown in Fig. 1(a). The distance of neighboring nodes along the hexagon is $100 \mathrm{~m}$. Each node is equipped with two radios, and each radio has two transmission power levels, which result in transmission ranges of 180 and $120 \mathrm{~m}$, respectively. For simplicity, we assume that the interference range of each radio is almost equal to

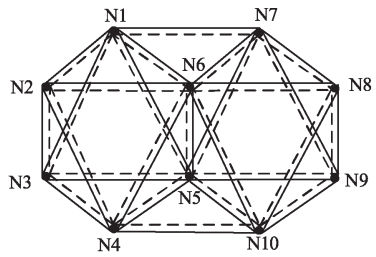

(a)

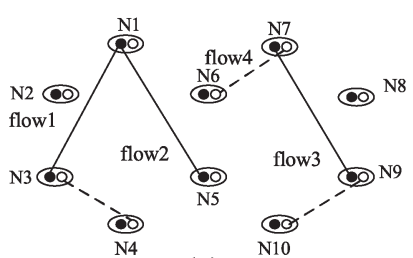

(c)

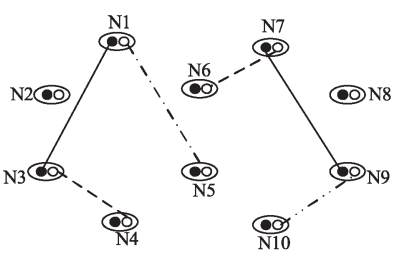

(e)

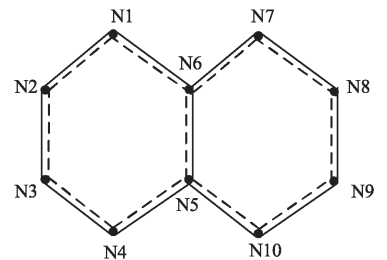

(b)

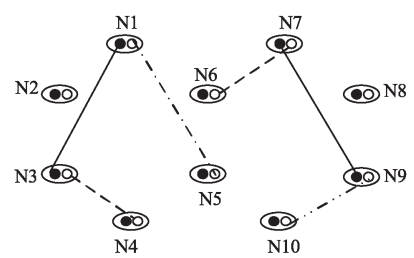

(d)

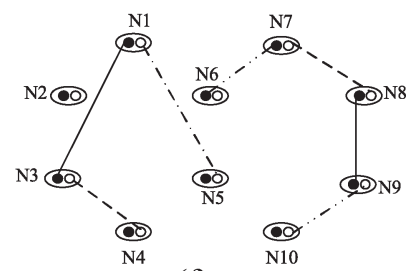

(f)

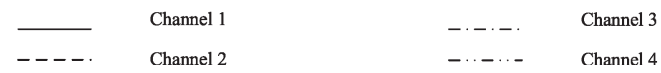

Fig. 1. Motivating scenario for joint topology control and routing.

its transmission range in this specific scenario. There are, in total, four orthogonal channels available. Assume that, initially, the first channel is assigned to the first radio, whereas the second channel is assigned to the second radio for all the nodes. Suppose that we tune the transmission power of each radio to the range of $180 \mathrm{~m}$. Then, the network topology is as illustrated in Fig. 1(a). Similarly, the network topology with a transmission range of $120 \mathrm{~m}$ is illustrated in Fig. 1(b). Each neighboring node pair has two virtual links that are tuned on channels 1 and 2, respectively. For simplicity, we assume that all the links are free of transmission error, and the raw capacity of each link is $11 \mathrm{Mb} / \mathrm{s}$.

As shown in Fig. 1(c), there are four flows, which are named flow1 (from node $N_{1}$ to node $N_{4}$ ), flow2 (from node $N_{1}$ to node $N_{5}$ ), flow3 (from node $N_{7}$ to node $N_{10}$ ), and flow4 (from node $N_{6}$ to node $N_{7}$ ). Here, each node in the figure is composed of two small circles-the black one and the white one-which denote the two radios. According to the up-to-date routing protocol proposed in [7], the route chosen in multiradio network should satisfy higher channel diversity in route path and lower packet loss rate properties. In this way, the potential routes ${ }^{1}$ for the four flows may be $N_{1}(1) \stackrel{1}{\rightarrow} N_{3}(2) \stackrel{2}{\rightarrow} N_{4}, N_{1}(1) \stackrel{1}{\rightarrow} N_{4}$, $N_{7}(1) \stackrel{1}{\rightarrow} N_{9}(2) \stackrel{2}{\rightarrow} N_{10}$, and $N_{6}(2) \stackrel{2}{\rightarrow} N_{7}$, respectively, as illustrated in Fig. 1(c). In this configuration, on both channels 1 and 2, there are three cochannel links that interfere with each other. Assume that all the four flows transmit a packet at the

\footnotetext{
${ }^{1}$ In multiradio network, the route (path) for a flow should be defined as a sequence of node ID and radio interface pair instead of the node ID only. We utilize $N_{i}(j) \stackrel{k}{\rightarrow}$ to present the route for flows, where $N_{i}$ denotes the node ID, $j$ denotes the traffic outgoing radio interface number, and $k$ denotes the channel utilized on that link.
} 
peak link data rate through a rough calculation that neglects overhead cost. Then, the aggregated network throughput of the four flows is about $11 \times(1 / 3)+11 \times(1 / 3)+11 \times(1 / 3)+$ $11 \times(1 / 3)=14.7 \mathrm{Mb} / \mathrm{s}$ since each flow can only occupy onethird fraction of channel access time.

Then, with the above-selected routes, we apply channel assignment to improve the network performance. After switching the overloaded channel into a less used one, as proposed in [10], both nodes $N_{1}$ and $N_{5}$ have one radio tuned from channel 1 to channel 3. Moreover, nodes $N_{9}$ and $N_{10}$ have one radio tuned from channel 2 to channel 4. As shown in Fig. 1(d), the cochannel interference on channels 1 and 2 is alleviated in that the number of interfering links is reduced from three to two. Now, the aggregated network throughput for the four flows is $11 \times(1 / 2)+11+11 \times(1 / 2)+11 \times(1 / 2)=27.5 \mathrm{Mb} / \mathrm{s}$. Note that there are still two interfering links ${ }^{2}$ on channel 1 $\left(l_{N_{1} N_{3}}^{1}, l_{N_{7} N_{9}}^{1}\right)$ and channel $2\left(l_{N_{3} N_{4}}^{2}, l_{N_{6} N_{7}}^{2}\right)$, respectively.

Based on the above route and channel assignment, we further check whether power control can be utilized to improve the network performance. As shown in Fig. 1(e), we lower the transmission power level ${ }^{3}$ of the second radio on nodes $N_{3}$, $N_{4}, N_{6}$, and $N_{7}$ so that the transmission ranges of those radios are reduced from 180 to $120 \mathrm{~m}$. Now, the previously conflicting link $l_{N_{3} N_{4}}^{2}$ and $l_{N_{6} N_{7}}^{2}$ can concurrently transmit. Through simple power control, the aggregated throughput is up to $11 \times(1 / 2)+11+11+11 \times(1 / 2)=33 \mathrm{Mb} / \mathrm{s}$.

To go further, the ideal adjustment for the network, as illustrated in Fig. 1(f), is to reduce the transmission power of nodes $N_{7}, N_{9}, N_{6}$, and $N_{10}$ to the transmission range of $120 \mathrm{~m}$. The routing path for flow 3 changes to $N_{7}(2) \stackrel{2}{\rightarrow}$ $N_{8}(1) \stackrel{1}{\rightarrow} N_{9}(2) \stackrel{4}{\rightarrow} N_{10}$. The channels of nodes $N_{6}$ and $N_{7}$ are switched from channel 1 to channel 4 . In this way, all the links may concurrently transmit. The aggregate throughput can reach $11+11+11+11=44 \mathrm{Mb} / \mathrm{s}$.

To summarize, the above example demonstrates that considering only channel assignment and routing is not good enough. To fully reduce the cochannel interference and consequently achieve higher gain of network performance, the topology control and routing should be jointly considered to exploit not only channel diversity but also spatial reusability. Before we discuss this further, we first give a formalized statement of the problem we are trying to solve in this paper.

\section{Problem Statement}

In this section, we present the basic definitions and concepts that are used in system model and then give a formulation for the JTCR problem.

We model the wireless mesh network as an undirected graph $G=(V, E)$. Nodes set $V$ represents wireless nodes, whereas edges set $E$ represents the virtual links set between nodes. We assume that there are $L$ orthogonal channels available in the system denoted by $c_{1}, c_{2}, \ldots, c_{L}$. Without losing generality,

\footnotetext{
${ }^{2}$ The notation $l_{N_{i} N_{j}}^{c}$ is utilized to denote the link between nodes $N_{i}$ and $N_{j}$ on channel $c$.

${ }^{3}$ The power change is not evident in Fig. 1(d) since we only draw the active links for clarity in the motivating scenario.
}

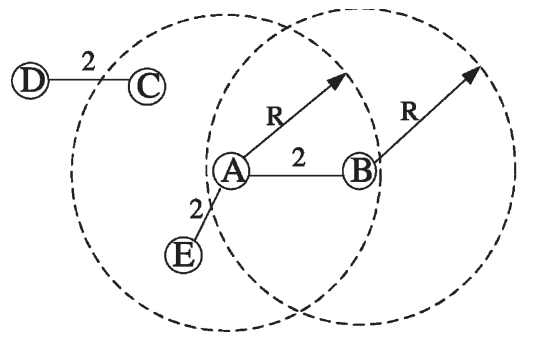

(a)

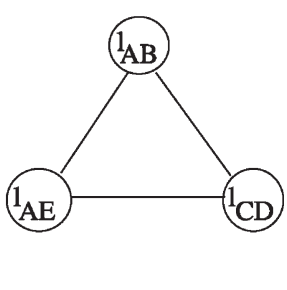

(b)
Fig. 2. Cochannel interference and conflict graph. (a) Network topology on channel 2. (b) Conflict graph on channel 2.

we assume that there are a total of $K$ types of radio in the network, which relate to $K$ channel sets $c s_{1}, c s_{2}, \ldots, c s_{K}$. For each channel set, we have $c s_{k} \subseteq\left\{c_{l}, l=1, \ldots, L\right\}$. Different radio is capable of selecting a working channel from the related channel set. For each node $u \in V$, it is equipped with one or more radio interfaces represented by $I(u)$, where $1 \leq I(u) \leq$ $K$. Thus, each wireless node $u$ is associated with an ordered set $A(u) \subseteq\{1,2, \ldots, L\}$ of $I(u)$ distinct channels.

We assume that a node can adjust the transmission power of each radio within a number of power levels $\left\{P_{1}, P_{2}, \ldots, P_{\max }\right\}$. Different power levels result in different transmission ranges $\left\{R_{1}, R_{2}, \ldots, R_{\max }\right\}$. Note that the power control is based on a per-radio scheme, that is, the transmission power of different radios on a node can be tuned to distinct power levels.

The traffic demands are assumed as a collection of $M$ end-to-end flows, i.e., $f_{m}=\left(s_{m}, d_{m}\right)$, where $s_{m}, d_{m} \in V$ and $m=1,2, \ldots, M . s_{m}$ and $d_{m}$, respectively, denote the source and destination node of flow $f_{m}$. Let the achieved throughput at the destination be $t_{m}$. Then, the overall throughput of a network is defined as $T=\sum_{1 \leq m \leq M} t_{m}$. Our target is to adjust the channel assignment, the power level of each radio, and the route for flows such that the network throughput is maximized.

In order for two nodes to communicate, it is required that the two nodes have a common channel that is assigned to their radio interfaces and be located within communication range of each other. For notational convenience, we will use $A(c)$ to denote the common working channels among $A(u)$ and $A(v)$. We denote by $R_{T}^{p, c}$ the transmission range of their radio, which resides on common channel $c$ with power level tuned on $p$, and by $d_{u, v}$ the distance between the nodes $u$ and $v$. An edge $e(u, v ; c) \in E$ if and only if $d_{u, v} \leq R_{T}^{p, c}, A(c) \neq \Phi$, and $c \in A(c)$.

Due to the broadcast nature of the radio interface, the success of a transmission is greatly influenced by the amount of multiple-access interference in neighborhood area. We denote by $R_{I}^{p, c}$ the interference range (which is typically $Q$ times of $R_{T}^{p, c}, Q \geq 1$ ) associated with each radio tuned on channel $c$ with power level $p$. Assume that $u, v, x, y$ are wireless nodes and link $e(u, v ; c) \in E, e^{\prime}(x, y ; c) \in E$ exist. If nodes $x$ or $y$ are within the interference range of $u$ or $v$, then transmission on link $e$ interferes with that of link $e^{\prime}$. This definition of the cochannel interference also includes the cases where the two links share a common node. As shown in Fig. 2(a), links 
$(A, B ; 2),(A, E ; 2)$, and $(C, D ; 2)$ interfere with each other on channel 2.

For the sake of clarity, we represent such a cochannel interference using a conflict graph. Corresponding to link $e(u, v ; c) \in E$ between nodes $u$ and $v$ on a specific channel $c$, the conflict graph of channel $c$ contains a node (denoted by $l_{u v}$ ). We place an edge between two nodes (for example, $l_{u v}$ and $\left.l_{x y}\right)$ in the conflict graph if the links $(u, v ; c)$ and $(x, y ; c)$ in the network interfere. Fig. 2(b) shows the contention graph on channel 2 for the network topology of Fig. 2(a).

After introducing the definitions and concepts, we give the problem formulation as follows.

\section{Given:}

- $V$, set of $n$ nodes;

- $L$, orthogonal channels available;

- $B$, bandwidth of each channel;

- $I(u)$ radio interfaces equipped for node $u$;

- $\left\{P_{1}, P_{2}, \ldots, P_{\max }\right\}$, power levels for each radio;

- $f_{m}$, traffic demand for each node pair $\left(s_{m}, d_{m}\right)$.

\section{Variables:}

- $e_{u, v ; c}$, Boolean variables, $e_{u, v ; c}=1$ if there is a link from node $u$ to node $v$ on channel $c$; otherwise, $e_{u, v ; c}=0$;

- $e_{u, v ; c}^{s_{m}, d_{m}}$, Boolean variables, $e_{u, v ; c}^{s_{m}, d_{m}}=1$ if the route from $s_{m}$ to $d_{m}$ goes through the link $(u, v ; c)$; otherwise, $e_{u, v ; c}^{s_{m}, d_{m}}=0$;

- $t_{u, v ; c}$, traffic load per unit time that was sent from node $u$ and achieved at node $v$ on channel $c$. Specifically, $t_{m}$ denotes the achieved throughput at the destination for traffic flow $f_{m}$.

\section{Optimize:}

$$
\max \sum_{1 \leq m \leq M} t_{m}
$$

\section{Output:}

- transmission power level $P_{u}^{i}$ for the $i$ th radio of node $u, 1 \leq i \leq I(u), \forall u \in V$;

- channel assignment scheme $A(u)$ for node $u$, $\forall u \in V$;

- delivery route $e_{u, v ; c}^{s_{m}, d_{m}}$ for flows, $\forall u, v \in V$.

\section{Constraints:}

— Topology constraints

$$
e_{u, v ; c}=e_{v, u ; c}, \quad \forall u, v \in V
$$

- Power constraints

$$
R_{T}^{p, c}(u) \geq d_{u, v} * e_{u, v ; c}, \quad \forall u, v \in V
$$

- Bandwidth constraints ${ }^{4}$

$$
\begin{array}{r}
\sum_{1 \leq m \leq M} \sum_{i o r j \in D\left(u, R_{I}^{p, c}\right)}\left(e_{i, j ; c}^{s_{m}, d_{m}} * t_{i, j ; c}+e_{j, i ; c}^{s_{m}, d_{m}} * t_{j, i ; c}\right) \\
\leq B, \quad \forall u \in V
\end{array}
$$

\footnotetext{
${ }^{4} D\left(u, R_{I}^{p, c}\right)$ denotes the set of nodes that are located within the interference range of node $u$ on channel $c$.
}

— Route constraints

$$
e_{u, v ; c}^{s_{m}, d_{m}} \leq e_{u, v ; c}, \quad \forall u, v \in V
$$

- Radio constraints

$\left\|\left\{c \mid e_{u, v ; c}=1,1 \leq c \leq L, \forall v \in V\right\}\right\| \leq I(u), \quad \forall u \in V$

- Constraint (2) ensures that each edge in our network corresponds to two directed links.

- Constraint (3) determines the allowable transmission power level for a radio on channel $c$.

- Constraint (4) ensures that the total transmission and reception of packets at a node's interference range on channel $c$ do not exceed the bandwidth capacity of this channel. The two terms of inequality (5) represent all the ingoing and outgoing traffics, respectively, on cochannel links that are within node $u$ 's interference range.

- Constraint (5) ensures the validity of the route ${ }^{5}$ for each node pair.

- Constraint (6) ensures that node $u$ who has $I(u)$ radios can be assigned at most $I(u)$ channels from $1 \leq c \leq L$.

From the problem formulation, we can see that the cochannel interference is a major obstacle for the improvement of network throughput. Therefore, our target of maximizing the total throughput that is achieved by all flows can be alternatively regarded as reducing the cochannel interference. Since the JTCR problem relates with many complex variables and is generally NP-hard, in this paper, we aim to design a distributed heuristic algorithm that adaptively adjusts the topology and routing to minimize the cochannel interference. However, the distributed algorithm leads to new challenges. First, a quantitative metric is necessary for estimating the channel conditions and determining whether an adjustment is needed. Furthermore, since we may have various adjustment candidates that represent specified combined solutions of channel assignment, power adjustment, and route change, a node should be able to evaluate which candidate is the best by the aid of this metric. We will define such a metric in the next section.

\section{Performance Metric ECATM}

Generally speaking, both the channel diversity and the spatial reusability affect the network performance in multiradio multichannel wireless mesh network. In our past work [10], the channel cost metric $(C C M)$ is proposed that considers the channel diversity while neglecting the effect of spatial reusability. Based on $C C M$, in this paper, we develop a new metric called ECATM to reflect also the spatial reusability characteristic. In this section, we will first briefly introduce the $C C M$ metric. Then, we will discuss the significance of spatial reusability by a simple example and illustrate how to estimate it in the metric calculation. Finally, we will give a closed-form expression of the proposed metric, i.e., ECATM.

\footnotetext{
${ }^{5}$ Only single path routing is considered. Multipath routing is beyond the scope of this paper.
} 


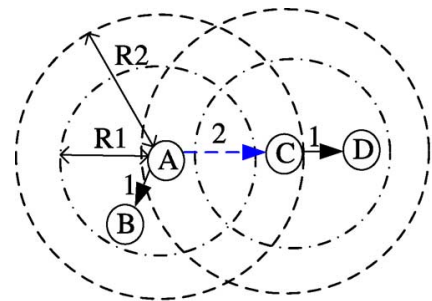

(a) (c)

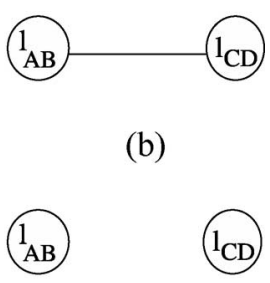

Fig. 3. Spatial reuse characteristic in metric design. (a) Network topology with four nodes and three links. (b) Conflict graph on channel 1 with a higher power level. (c) Conflict graph on channel 1 with a lower power level.

$C C M$ represents the cost on channel time that is observed at node $i$ to support the current traffic. It is defined as

$$
C C M_{i}=\sum_{c} \sum_{l} r_{l}^{c} E T T_{l}^{c} F^{c}
$$

where $c$ denotes the available channels, and $l$ denotes the cochannel links that lie in the interference range of a specific node $i$.

In this equation, $r_{l}^{c}$ denotes the data traffic rate (in packets per second) for link $l$ on channel $c$, and $E T T_{l}^{c}$ represents the expected transmission time for one packet (with an averaged packet length for link $l$ ) on link $l$. Therefore, the value of $r_{l}^{c} E T T_{l}^{c}$ represents the expected transmission time for the current traffic on link $l$.

The ETT [7] of a link $l$ on channel $c$ is defined as

$$
E T T_{l}^{c}=E T X_{l}^{c} * \frac{S}{B}
$$

where $E T X_{l}^{c}$ is the expected number of transmission attempts (including retransmissions) that is required to transmit a packet, as defined in [12]. $S$ is the average packet size, and $B$ is the bandwidth of the link.

In (7), $F^{c}$ denotes the total air time utilization on channel $c$ that is observed at node $i$, which is defined as

$$
F^{c}=\sum_{l} r_{l}^{c} E T T_{l}^{c}
$$

Therefore, $C C M$ is essentially the summation of total ETT in a time unit weighted by the channel utilization $F^{c}$ [10]. A smaller value of the $C C M$ metric implies less occupation of channel air time and diverse channel allocation. For more details of $C C M$, see [10].

As we have emphasized before, besides channel diversity, spatial reuse can be utilized to further improve the network performance. We use a simple example to demonstrate it. Consider a network topology as shown in Fig. 3(a); each node is equipped with two radios, and each radio has two power levels that correspond to the transmission range of $\mathrm{R} 2$ and $\mathrm{R} 1$, respectively. Assume that there are two flows on the three links-flow1 on path $A(2) \stackrel{2}{\rightarrow} C(1) \stackrel{1}{\rightarrow} D$ and flow2 on path $A(1) \stackrel{1}{\rightarrow} B$. Assume that the achieved data rate and the expected transmission time of each packet for the three links are equal, which are denoted by $r$ and $E T T$, respectively. By adjusting the transmission power on channel 1 , we get two possible candidates-one with transmission range $\mathrm{R} 2$ and the other one with transmission range $\mathrm{R} 1$. The conflict graph of channel 1 for both candidates is illustrated in Fig. 3(b) and (c), respectively. With a lower transmission power level, channel 1 is spatially reused so that previously interfering links $l_{A B}^{1}$ and $l_{C D}^{1}$ can concurrently transmit.

It is obvious that the candidate with transmission range $\mathrm{R} 1$ on channel 1 is better than that of R2. However, without considering the spatial reusability in $C C M$ metric design, we have equal $C C M$ values $\left(C C M=\sum_{c} \sum_{l} r_{l}^{c} E T T_{l}^{c} F^{c}=\right.$ $2 r E T T * 2 r E T T+r E T T * r E T T=5 r^{2} E T T^{2}$ ) for the two candidates. In order to distinguish them, we need to design a new metric that can reflect both channel diversity and spatial reusability.

Before we discuss the new metric, we first clarify a semantic modification in $C C M$. In the definition of $C C M$, links and traffic load information within the interference range of a node are collected and calculated. Since the interference range changes with the distance between different communicating node pairs, in the protocol design, two-hop range is employed to approximately represent the interference range $R_{I}^{P}$ for a radio with a power level $P$. Considering that the interference range may change with the adjustment of transmission power levels, conservatively, we fix the interference range to the one with the maximal power level $R_{I}^{P_{\max }}$. Correspondingly, in our protocol design, the two-hop range approximation of the interference range is fixed to the two-hop range with maximal power level.

According to the intuition that is obtained from the above example, we propose a new performance evaluation metric $E C A T M$ defined as

$$
E C A T M_{i}=\sum_{c} \sum_{l} r_{l}^{c} E T T_{l}^{c} F^{c} / S R^{c}
$$

where $c$ represents the available channels, $l$ denotes the cochannel links that lie in the interference range $R_{I}^{P_{\max }}$ of a specific node $i$, and $S R^{c}$ denotes the channel reuse factor on channel $c$. $E C A T M$ represents the aggregated equivalent channel air time for potential candidates and acts as an indicator for the network performance observed at node $i$.

Now, we discuss how to estimate the spatial reuse factor for a given channel. The precise calculation of channel air time consumption under the constraint of spatial reuse is very complicated. It depends on the independent sets calculation and a specific scheduling scheme. Since we only want to compare the relative goodness for different candidates rather than get the exact value of the spatial reuse factor, we simplify this problem to find noninterfering link groups. A noninterfering link group denotes that none of the links in one group interferes with the links in another group, whereas the links in the same group may interfere with each other.

As shown in Fig. 4(a), in node D's two-hop area, there are five active links with traffic load $r$ on channel 1 . The corresponding conflict graph is illustrated in Fig. 4(b). From 

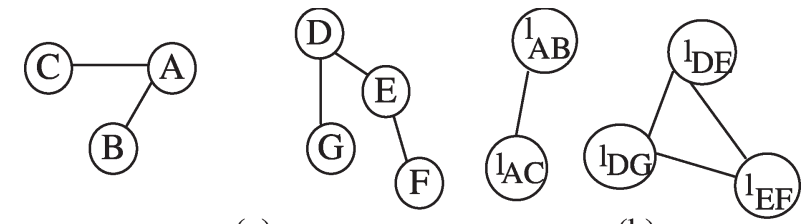

(a)

(b)

Fig. 4. Noninterfering link group for spatial reuse. (a) Connectivity graph with traffic flow on channel 1 in node $D$ 's two-hop area. (b) Corresponding conflict graph.

the conflict graph, we can see that the five cochannel links may be divided into two noninterfering groups, i.e., $\left\{l_{A B}^{1}, l_{A C}^{1}\right\}$ and $\left\{l_{D E}^{1}, l_{D G}^{1}, l_{E F}^{1}\right\}$. These two groups spatially reuse channel 1 and do not interfere with each other. Under the assumption of spatial reuse, the total channel air time needed for channel 1 now becomes the sum of each group, which is up to $2 r E T T * 2 r E T T+3 r E T T * 3 r E T T=13 r^{2} E T T^{2}$. However, if we ignore the impact of spatial reuse, the channel air time needed for channel 1 in node $D$ 's two-hop area is equal to $\sum_{l} r_{l}^{c} E T T_{l}^{c} F^{c}=25 r^{2} E T T^{2}$. Intuitively, the spatial reuse factor is the ratio between the channel air time with and without considering spatial reusability (25/13 in this case). Assume that there are $G$ noninterfering groups. We then formalize the spatial reuse factor $S R^{c}$ as

$$
S R^{c}=\frac{\sum_{l} r_{l}^{c} E T T_{l}^{c} F^{c}}{\sum_{g} \sum_{l} r_{l}^{c} E T T_{l}^{c} F_{g}^{c}}
$$

where $1 \leq g \leq G$, and $F_{g}^{c}$ denotes the channel utilization in a specific group.

Similarly, we derive the channel reuse factor on channel 1 for the two candidates that are shown in Fig. 3(b) and (c), which is 1 and 2, respectively. Consequently, the weighted sum $\sum_{c} \sum_{l} r_{l}^{c} E T T_{l}^{c} F^{c} / S R^{c}$ is $5 r^{2} E T T^{2}$ and $3 r^{2} E T T^{2}$, respectively. The performance obtained by these two candidates can now be distinguished correctly. In short, ECATM favors candidates that result in good link quality, diverse channel allocation, and a higher level of channel spatial reuse. It is consistent with our design goals.

\section{Protocol Design}

In this section, we elaborate on the distributed JTCR algorithm. The key idea is to select the JTCR candidate that results in the smallest ECATM value and then perform the corresponding negotiation and adjustment. It is assumed that each node knows the traffic and link status on all channels within the two-hop neighbor range. This is a rather practical assumption since only one-hop information exchange by broadcast is needed. Moreover, each node has the neighbor node information under different power levels. With this information, each node may estimate the value of ECATM and make the corresponding decision in a distributed manner.

There are many different choices for adjustment. A JTCR adjustment candidate denotes any specific combined solution of channel switching, power adjustment, and route change. A

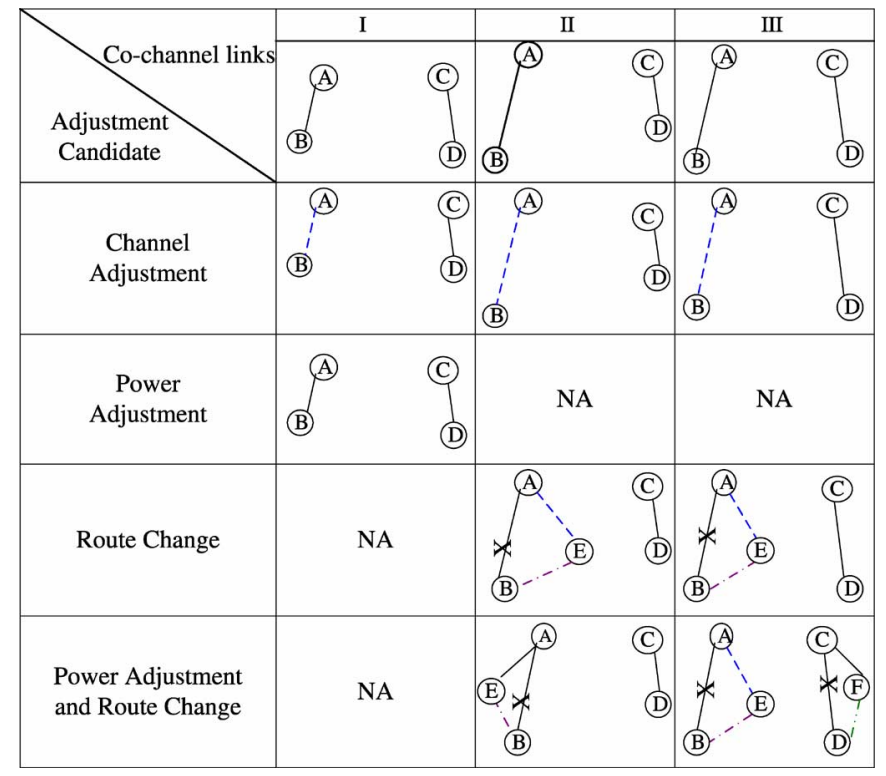

Fig. 5. Possible candidate.

candidate is called feasible only when it observes the following three constraints.

1) The new candidate should maintain the current throughput that is achieved by each flow. Given the negotiation overhead for the adjustment, the performance gains in terms of ECATM should be larger than a threshold.

2) Considering the potential risk of network partition, the adjustment candidate should preserve the network connectivity.

3) The adjustment of power levels should not cause asymmetric links, which exacerbate the hidden terminal problem [13].

\section{A. Candidate Set}

We first discuss the candidate set for possible adjustment. The candidate set searching process is essentially trying to find the opportunity for exploiting channel diversity and spatial reusability. Generally speaking, the cochannel interfering links can be divided into three types. As shown in Fig. 5, type I denotes that the two interfering links that are located in node $A$ 's two-hop area are reachable through a power level lower than the current one. The possible adjustment candidate can be channel adjustment (switch one link to another channel) and power adjustment (reduce the transmission power levels of $A, B, C$, and $D$ so as to exploit spatial reuse). Type II denotes that one link is only reachable from the current power level, whereas the other one is reachable through a lower power level. In this case, we have three possible adjustment candidates - channel adjustment, route change [find a two-hop alternative path $(A \rightarrow E \rightarrow B)$ to replace link $l_{A B}$ ], or power adjustment plus route change. In type II, since reducing the power level of $A$ may break the long link $l_{A B}$, we have to change the route, that is, find a two-hop alternative path to compensate it. When both of the interfering links are only reachable through the current power level as classified in type III, 
channel adjustment, route change, and power adjustment plus route change are the possible adjustment candidates, as shown in Fig. 5. In this case, a separated power adjustment is not allowable since the two long links are lost without two-hop path compensation.

The adjustment candidate selection process is triggered only when a node detects the overloaded channel condition that it concerns. ${ }^{6}$ It checks the feasibility of possible candidates on the most overloaded channel under the premise that it can directly benefit from the adjustment. Therefore, it actually selects from only a few possible choices and does not cause the cardinality explosion of the candidate sets.

\section{B. Performance Assurance}

When selecting an adjustment candidate, we have to guarantee that the new candidate improves the network throughput. Moreover, we expect that the new candidate will not cause any throughput degradation of other channels. This goal can be interpreted in the following.

1) The overall network performance should be improved. It is required for an adjustment candidate that the $E C A T M$ value after adjustment be less than the original one. Considering the adjustment and negotiation overhead, the gains in terms of ECATM should be larger than a given threshold (GThreshold) to compensate the extra overhead.

$$
\frac{E C A T M_{\text {original }}}{E C A T M_{\text {after }}} \geq \text { GThreshold } .
$$

2) Since some flows may be switched to other channels, it should be ensured that previously nonoverloaded channels do not become overloaded after adjustment. For a given channel $c$, suppose that there are $G$ noninterfering groups that are observed at node $i$. Only when the channel utilization $F_{g}^{c}$ for each group is less than a certain threshold (CThreshold) can we say that channel $c$ is not overloaded.

$$
F_{g}^{c} \leq \text { CThreshold, } 1 \leq g \leq G .
$$

In our implementation, GThreshold is set to 1.2, that is, the network performance after adjustment in terms of ECATM should at least be $20 \%$ better than the original one. We set $C$ Threshold $=1$, ignoring the potential time overlap within the noninterfering group. Therefore, it is a conservative but sufficient condition.

Only when the two conditions are satisfied can we say that the current throughput of each flow can be maintained by the new adjustment candidate.

\section{Network Connectivity and Link Symmetry}

Now, we discuss the connectivity and link symmetry issues. The reduction of power level and channel adjustment of a node may lose several connecting links with neighbor nodes or may

\footnotetext{
${ }^{6}$ It has outgoing or incoming traffic resides on this overloaded channel.
}

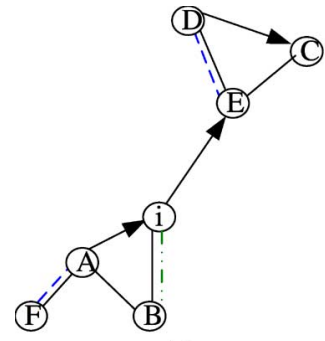

(a)

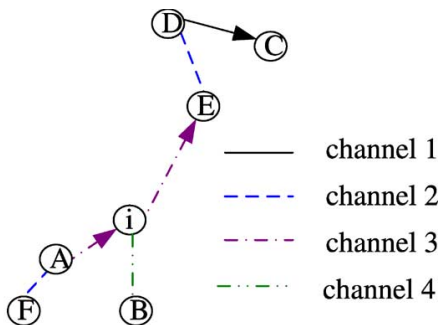

(b)
Fig. 6. Connectivity in channel assignment. (a) Initial network topology. (b) After channel adjustment of node $i$.

even cause some nodes to be isolated from network. Therefore, when we choose an adjustment candidate, we have to ensure that the adjustment will not result in network partition. Note that under a multiradio multichannel environment, two neighbor nodes may have more than one pair of radios connected. Two nodes are connected if they have at least one pair of radios connected. In this paper, we only require two-hop spanner connectivity, that is, a node pair is regarded as connected if they can reach each other directly or through a two-hop path. This less-restricted requirement of the network connectivity increases the flexibility and provides more opportunity for a feasible adjustment.

Among the three basic adjustment types (channel adjustment, power adjustment, and route change), only channel and power adjustments may affect the network connectivity. Since each node independently makes a decision, it may cause inconsistency and confusion when two nearby nodes simultaneously adjust their topology. Thus, it is required that both the channel adjustment and the power adjustment should guarantee the exclusiveness of adjustment in the node's interference area. Under this premise, each node may locally make adjustment decisions without considering the disturbance of neighboring nodes. The solution for the exclusiveness will be discussed in Section V-E. Under this premise, each node may locally make adjustment decisions without considering the disturbance of other nodes.

1) Channel Adjustment: The channel adjustment of a node $i$ has different impacts on its neighborhood nodes. We use a simple example, as shown in Fig. 6(a), to illustrate this. Assume that there is one flow $\mathrm{f} 1$ from nodes $A$ to $E$ on path $A(1) \stackrel{1}{\rightarrow} i(1) \stackrel{1}{\rightarrow} E$. Suppose that node $i$ finds that channel 1 is overloaded and wants to tune the channel from 1 to 3 , which is currently the least used channel around node $i$. For those neighboring nodes that have two radios connected with node $i$, for example, node $B$, the channel adjustment does not influence their connectivity since there is another link from a different radio that connects them. Therefore, our focus is the connectivity issue of neighbor node pairs that have only one common radio between them.

The links that previously connect node $i$ and neighbor nodes will be broken due to the channel switching of node $i$ if they have only one common radio. For those neighbor nodes that have active links (there is a traffic flow on the link) that are connected with node $i$, for example, nodes $E$ and $A$, they have to correspondingly switch their channel to keep the connectivity 


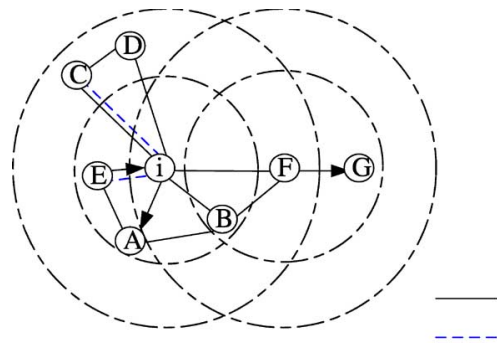

(a)

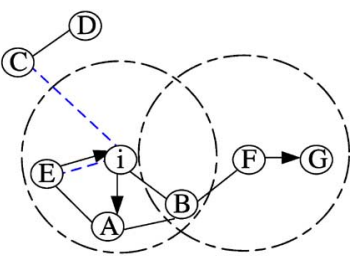

channel 1

channel 2

\section{(b)}

Fig. 7. Connectivity in power adjustment. (a) Network topology with a higher power level. (b) Network topology after power adjustment.

and the traffic flow. For an inactive link, we only need to ensure that the alternative two-hop path exists. The neighbor node $C$ belongs to this neighbor category of node $E$ since there is a two-hop path $[E(2) \stackrel{2}{\rightarrow} D(1) \stackrel{1}{\rightarrow} C]$. If no two-hop path exists, the channel adjustment is not allowable. The resulting topology after channel adjustment is illustrated in Fig. 6(b).

Besides the connectivity issue, the channel adjustment suffers from the propagation problem. As aforementioned, neighbor nodes $E$ and $A$, as shown in Fig. 6(a), have to switch their radios from channel 1 to channel 3 to keep the connectivity with node $i$. Suppose that the link between nodes $C$ and $E$ is an active link. Then, node $C$ has to switch its radio from channel 1 to channel 3 , together with nodes $E, A$, and $i$. Furthermore, if node $C$ has its neighbor that needs to switch channel, this channel adjustment may propagate along a chain of nodes or even affect the whole network. In order to bound the effect of channel switching in the local area, we impose a restriction on the neighbor node set to ensure that the adjustment does not propagate further; that is, the neighbors of $\{A, E\}$ that should also switch channel have to be confined in the channel switching neighbor node set of $i$. Otherwise, the adjustment candidate is not feasible. In this way, the channel adjustment is limited to a one-hop area. The detailed procedure for the connectivity check and propagation detection is discussed in Appendix A.

2) Power Adjustment: The connectivity issue in power adjustment is similar to channel adjustment in the sense that both of them need to keep the connectivity and avoid chain propagation. We also give an example to illustrate the connectivity and link symmetry issues in the power adjustment. As shown in Fig. 7(a), the smaller circle denotes the transmission range of a lower power level, whereas the larger one denotes that of a higher power level. Assume initially that all the radios are tuned on a higher power level. There are three traffic flows- $\mathrm{f} 1(i(1) \stackrel{1}{\rightarrow} A), \mathrm{f} 2(E(1) \stackrel{1}{\rightarrow} i)$, and $\mathrm{f} 3(F(1) \stackrel{1}{\rightarrow} G)$.

In general, the reduction of node $i$ 's power level only affects the connectivity with the neighbor nodes that are reachable only through a higher power level. For neighbor nodes $A$, $B$, and $E$, the power adjustment of node $i$ does not affect their connectivity since they are reachable with a lower power level. The connectivity can also be kept if node pairs have two common radios connected (node $C$ for example) or if twohop paths exist (for example, nodes $D$ and $F$ ) between these neighboring nodes. If neighbor nodes that do not belong to the above categories exist, the power adjustment will break the connectivity and, therefore, is not feasible.

Furthermore, in order to avoid asymmetric links, the power adjustment should be performed in a unit of a noninterfering link group, that is, all the nodes within the noninterfering link group should adjust their power together. Neighbor nodes of $i$ that have active links that are connected with node $i$ have to adjust their power level accordingly, for example, nodes $A$ and $E$. As for node $F$, it is located within the transmission range of node $i$ with a higher power level. In order to avoid hidden terminal, node $F$ should reduce its power level as well. Since node $G$ has an active link that is connected with node $F$, it should adjust its transmission power as well. When we reduce the power level of node set $\{F, G\}$ together with node set $\{i, A, E\}$, the previously interfering link group $\left\{l_{i E}, l_{i A}, l_{F G}\right\}$ splits into two noninterfering link groups (one is $\left\{l_{i E}, l_{i A}\right\}$; the other one is $\left\{l_{F G}\right\}$ ). Therefore, it is a possible candidate for power adjustment with performance gains. The network topology after the power adjustment of node $i$ is shown in Fig. 7(b).

Power adjustment may also bring about chain propagation. For example, if the node set $\{G, A, E\}$ also has other neighbors that need to adjust power, the propagations of power adjustment would happen. Similar to the scheme used in the channel adjustment, we should guarantee that the neighbors of node $\{A, E\}$ that should adjust power are confined to the neighbor set of node $i$, whereas the neighbors of node $\{G\}$ that should adjust power are confined to the neighbor set of $F$. Any power adjustment candidate that cannot satisfy the above restrictions is not a feasible candidate. The detailed procedure for the power adjustment connectivity check is discussed in Appendix B.

\section{Distributed Algorithm}

In this section, we describe the detailed algorithm for JTCR. Generally speaking, the algorithm performs the following steps in the given order.

- Each node periodically checks the channel condition. It calculates the $F_{g}^{c}$ value for each noninterfering group on each channel and checks if it is larger than the given threshold $C$ Threshold $=1$. If channels on which this node works overload, and this node is involved in the most overloaded noninterfering group, try to find the least used channel, and then, execute the following steps.

- Find available adjustment candidates, and check their feasibility. For each candidate, check first whether the corresponding adjustment will break the network connectivity; then, classify those neighbors into node sets of different adjustment type as follows: 1) Reduce transmission power; 2) change the route; 3 ) change the interface of traffic flow; and 4) switch the channel. These node sets may have overlaps; for example, some neighbors are required to adjust the power level as well as change the route path of traffic flow. Finally, calculate the result ECATM after the potential adjustment, and check if the performance assurance conditions can be satisfied-if yes, it is a feasible adjustment candidate; otherwise, this adjustment candidate should be abandoned. 


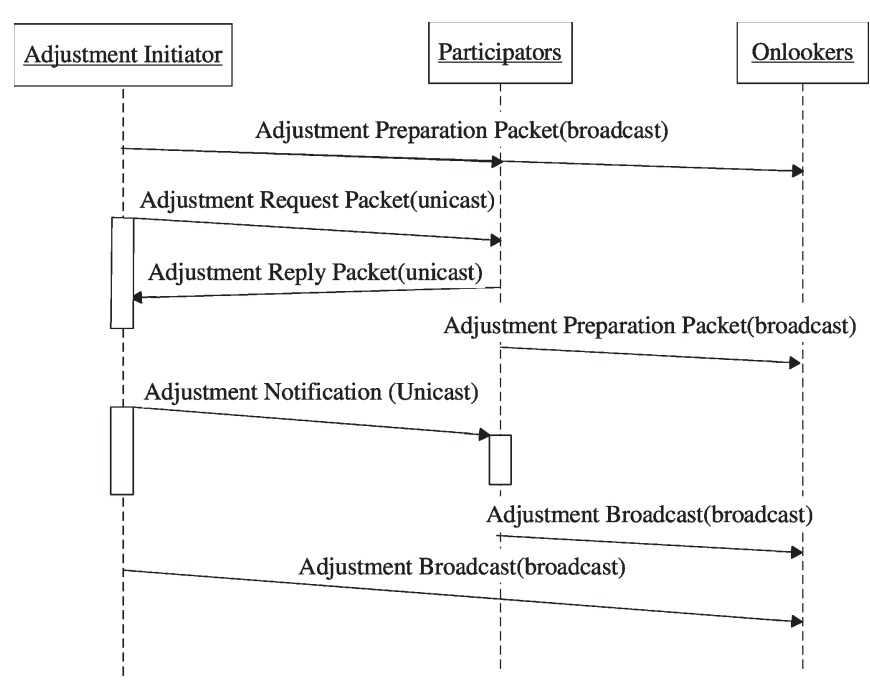

Fig. 8. Negotiation mechanism.

- Compare these feasible candidates, and select the one that has the smallest ECATM value. Then, begin the negotiation process.

A more detailed description of the algorithm is given in Appendix C.

\section{E. Negotiation Mechanism}

The joint adjustment of topology and routing usually needs the cooperation of other nodes. Therefore, negotiation mechanism is indispensable to make sure all the affected nodes consent to the adjustment. According to their roles in the negotiation, nodes can be divided into three categories-initiator, participator, and onlooker. An initiator denotes a node that first initiates the negotiation process. Participators refer to neighbors of the initiator that should also make the corresponding adjustments, for example, reducing the power or switching the channel. All the other neighbor nodes of the initiator and the participators belong to the onlookers. As shown in Fig. 8, the negotiation procedure is listed as follows.

1) The initiator sends an adjustment preparation broadcast packet to notify the participator and onlooker nodes, which, consequently, reschedule their channel check timers to ensure that no new negotiation happens.

2) The initiator sends an adjustment request that includes the adjustment information of all affected nodes to the participator nodes and then starts the reply-waiting timer and waits for their reply.

3) After receiving the adjustment request, participators check the feasibility of the required adjustment and then send an adjustment reply packet to the initiator about their decisions. Moreover, the participators broadcast an adjustment preparation broadcast packet to notify their onlooker with the same purpose as described in step 1 .

4) When the reply-waiting timer expires, the initiator checks the adjustment replies to see if all the participators agree with the adjustment, If yes, the initiator sends an adjustment notification to all the participators and starts the notification timer. If not, the negotiation fails.

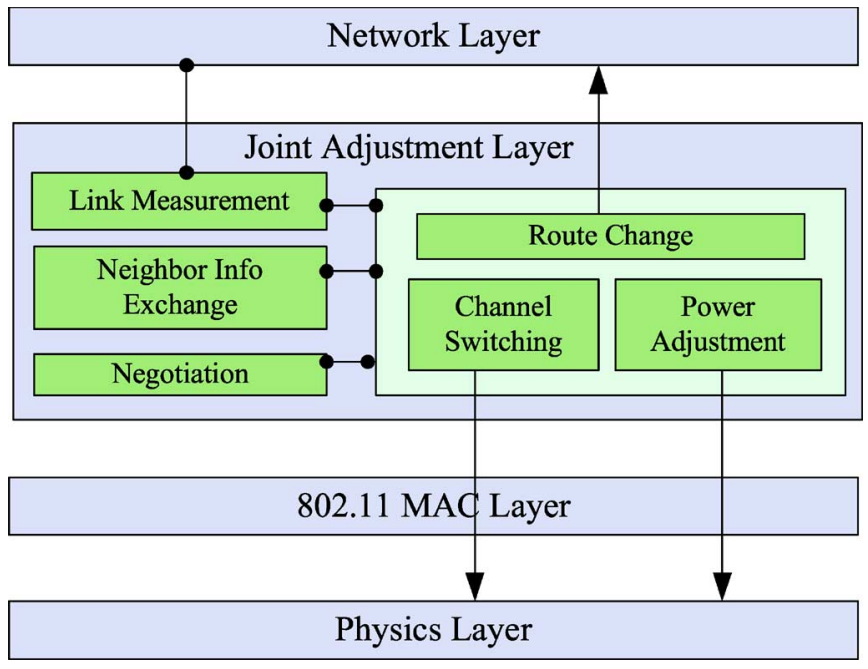

Fig. 9. Proposed architecture.

5) When the notification timers expire, both the initiator and the participators make the corresponding adjustments. Until now, the adjustments really take effect. Finally, the initiator and the participators broadcast their adjustment information through which onlookers could update their two-hop neighbor information on a timely basis.

In order to avoid packet loss during the negotiation, adjustment request, adjustment reply, and adjustment notification packet are sequentially sent three times to ensure reliability. If the adjustment of a node is related with route change, for example, node $i$, there is an additional process of route negotiation. When it receives a packet with obsolete path information, node $i$ tunnels the packet to the up-to-date route. Meanwhile, it sends a route update packet to the traffic source. The traffic source node updates its cache and then sends a route accept packet back to node $i$. Only when all route accept packets are received does node $i$ remove the route change information.

\section{Protocol Implementation}

In this section, we describe the implementation experiences of the JTCR protocol. We add a joint adjustment layer between MAC and network layer to implement the protocol. Fig. 9 outlines the proposed architecture. It interacts with the routing and physics layer to realize the route change and the channel and power adjustments. The joint adjustment layer is not tied to any specific routing algorithm. It can work with different routings that are applicable to a multiradio multichannel environment. For evaluation purposes, we implement a link-quality-aware routing protocol in the network layer based on dynamic source routing [14] and utilize the routing metric proposed in [7]. The interaction with network layer focuses on the route change adjustment. There are two types of route changes. One is only the traffic outgoing interface adjustment with the whole path unchanged, whereas the other one replaces the original link with a two-hop path. No modifications are required to the IEEE 802.11 MAC layer.

The joint adjustment layer consists of three modules-link measurement, neighbor information exchange, and negotiation. 
Among them, the link measurement module is utilized to collect the link status information, for example, packet loss ratio and traffic load. The estimation of link packet loss ratio is based on the approach introduced in [12]. Concerning the traffic load information, a time sliding window method [15] is employed to measure the traffic rate on a link. In the neighbor information exchange module, each node broadcasts a "Hello" message several times with different transmission power levels sequentially at the mesh network setup phase. The specific power level of the source and the previously inferred neighbor information from the received "Hello" message are piggybacked in the "Hello" message. Through this information exchange, the node can get the basic neighbor information under different power levels at the beginning. After that, the node periodically broadcasts the traffic rate and the packet loss ratio for each active link to/from it, together with the channel and power information to its neighbors. The information of its corresponding neighbor nodes is also piggybacked in this packet. This way, the node can obtain the traffic and link status within the two-hop range. The negotiation module is implemented to coordinate nodes and complete the adjustment.

\section{Simulation Results}

To study the overall performance gains of the proposed JTCR algorithm, we perform an extensive simulation using NS-2. NS-2 is modified to support multiradio interface on each wireless node and multiple orthogonal channels. The following are the default settings for the simulations. Each node is equipped with two radio interfaces. Each radio has two power levels that roughly correspond to transmission ranges of 120 and $180 \mathrm{~m}$. The carrier sense ranges are about 255 and $170 \mathrm{~m}$, respectively. Initially, the first radio works on channel 1 , whereas the second radio works on channel 2 . The data rate of all channels is set to $11 \mathrm{Mb} / \mathrm{s}$. All simulated data packets are preceded by a requestto-send/clear-to-send exchange. The adjustment algorithm is activated at about $20 \mathrm{~s}$ and checks the channel condition every $5 \mathrm{~s}$. For traffic rate measurement, the time sliding window size is $2 \mathrm{~s}$.

\section{A. Performance in Regular Topology}

We first look at the performance of the proposed approach in the regular topology, as illustrated in Fig. 1. Four constant bit rate (CBR) flows with equal traffic rate are set up between the given node pairs at time randomly chosen from [3] and [8]. There are four orthogonal channels available. Fig. 10 compares the aggregated flow throughput as a function of the aggregated offered traffic load under four scenarios-without adjustment, with channel and routing adjustment, with power and routing adjustment, and with our proposed joint adjustment. The result of without adjustment utilizes two channels and quantifies the performance of weighted cumulative expected transmission time (WCETT) metric [7]. The results in Fig. 10 show that with the proposed algorithm, the network throughput becomes two to three times that of the network without adjustment. Intuitively, the joint adjustment breaks the cochannel interfering link group into several interfering link groups, each residing on a different channel. An interfering link group is further

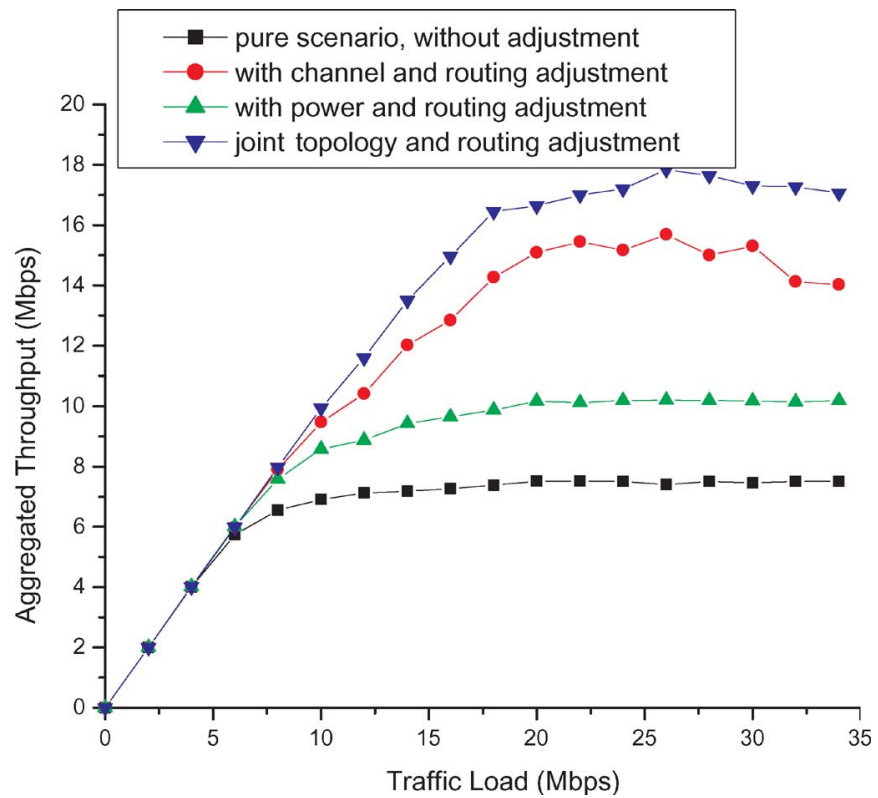

Fig. 10. Aggregated throughput in regular topology with varying traffic load.

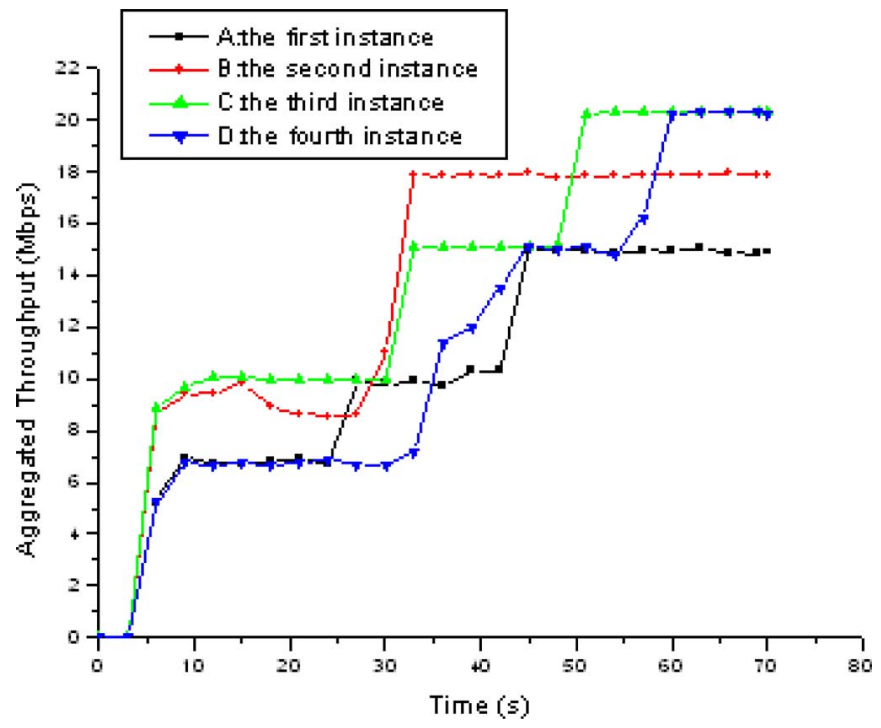

Fig. 11. Sampled simulation instances with joint adjustment.

spatially subdivided into smaller noninterfering link groups. By exploiting channel diversity and spatial reusability, cochannel interference is alleviated, which is the key reason for the throughput improvement.

The performance gains largely owe to the efficient utilization of new channels. Instead of previously two channels, four channels are employed through the distributed negotiation and adjustment. Compared with channel adjustment, power adjustment heavily depends on the underlying topology and traffic profile and, thus, does not perform as well as channel adjustment.

Fig. 11 shows the throughput change of four joint adjustment instances with respect to time. Due to the randomness of flow injection time, the link quality aware routing may choose different paths each time for the simulation, thus resulting in the distinctness of primitive throughput before adjustment. 


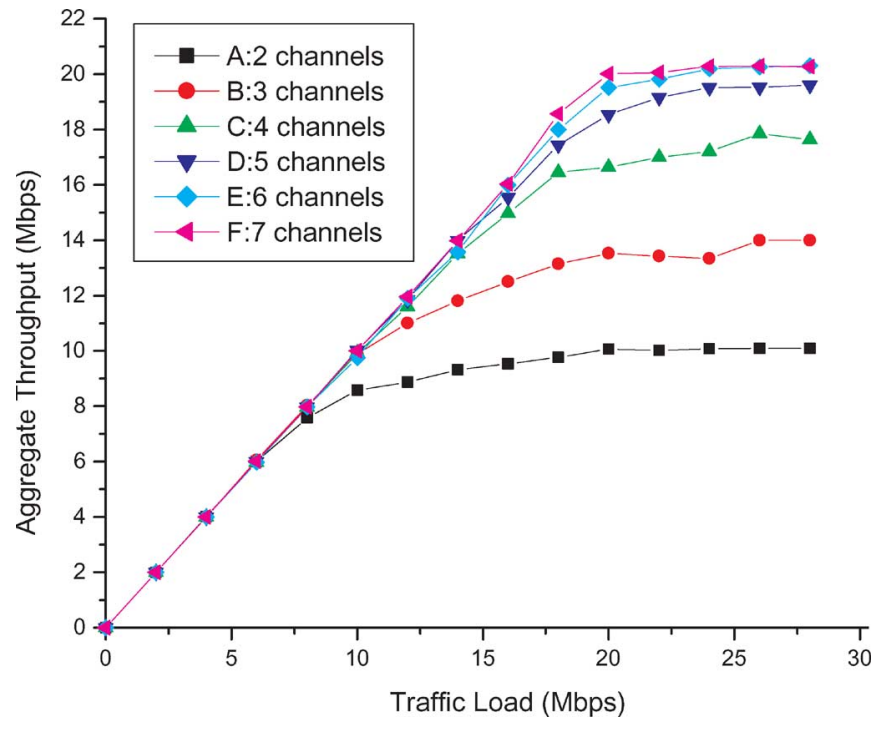

Fig. 12. Impact of increasing the number of available channels for regular topology.

For example, the initial throughput for instance $\mathrm{A}$ is about $10 \mathrm{Mb} / \mathrm{s}$ before adjustment, whereas instances $\mathrm{C}$ and $\mathrm{D}$ are only $6.8 \mathrm{Mb} / \mathrm{s}$. This kind of randomness also reflects in the subsequent adjustment. Since the node sequences that initiate the adjustment and the type of adjustment are not the same every time, we obtain rather diverse curves, as shown in Fig. 11. Sometimes, we get close to the optimal result, for example, instances $\mathrm{C}$ and $\mathrm{D}$. In other cases, we only obtain suboptimal improvement, for example, instance A. To avoid the influence of randomness, every curve in Fig. 10 is an average of all results for various route selections and adjustment sequences.

The performance of our algorithm has been evaluated with two, three, four, five, six, and seven orthogonal channels. The simulation demonstrates that the joint adjustment algorithm can effectively adapt itself with the number of available channels, as illustrated in Fig. 12. The result in Fig. 12 also suggests that increasing the channels does not help in infinitely improving the throughput. When the number of channels is six or more, we get similar curves with that of five channels. The reason is that each node has only two radio interfaces. Due to the restriction of radio interface number and the influence of channel dependence, the extra channels cannot be fully utilized.

\section{B. Random Topology}

We relax the regularity of node placement. Assume that 30 nodes are uniformly placed on a $750 \times 750 \mathrm{~m}$ area. Five CBR flows are set up between randomly selected node pairs. First, we evaluate the throughput improvement in random scenario. Since the aggregated throughput obtained depends on the topology, we normalize all results with the throughput obtained when only using the link quality aware routing on two channels without adjustment.

Fig. 13 compares the throughput of joint adjustment, channel and routing adjustment, or power and routing adjustment. Results are plotted for ten different random topologies. As we can see, the normalized throughput of joint adjustment with

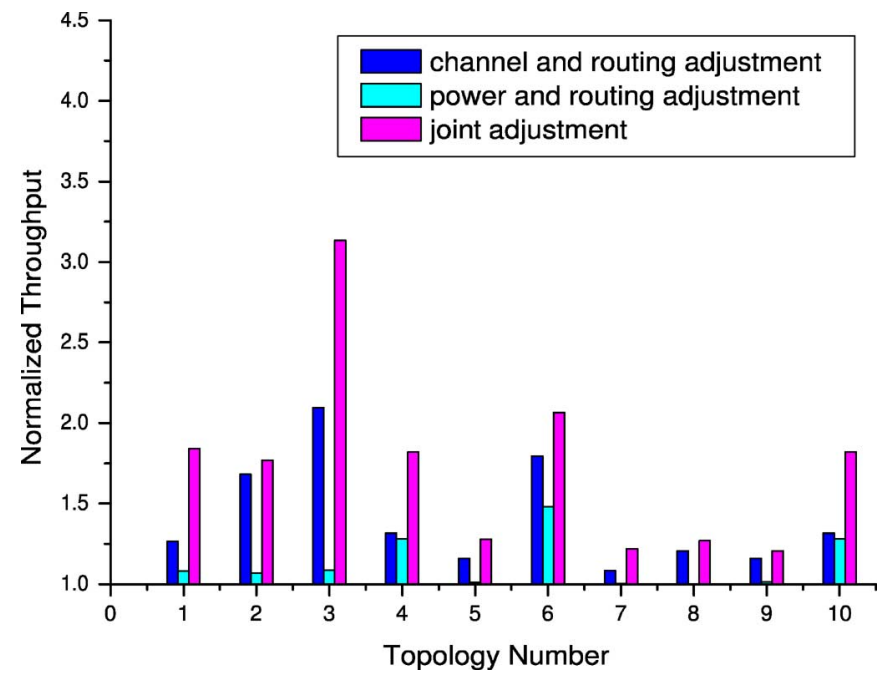

Fig. 13. Normalized throughput in random topologies.

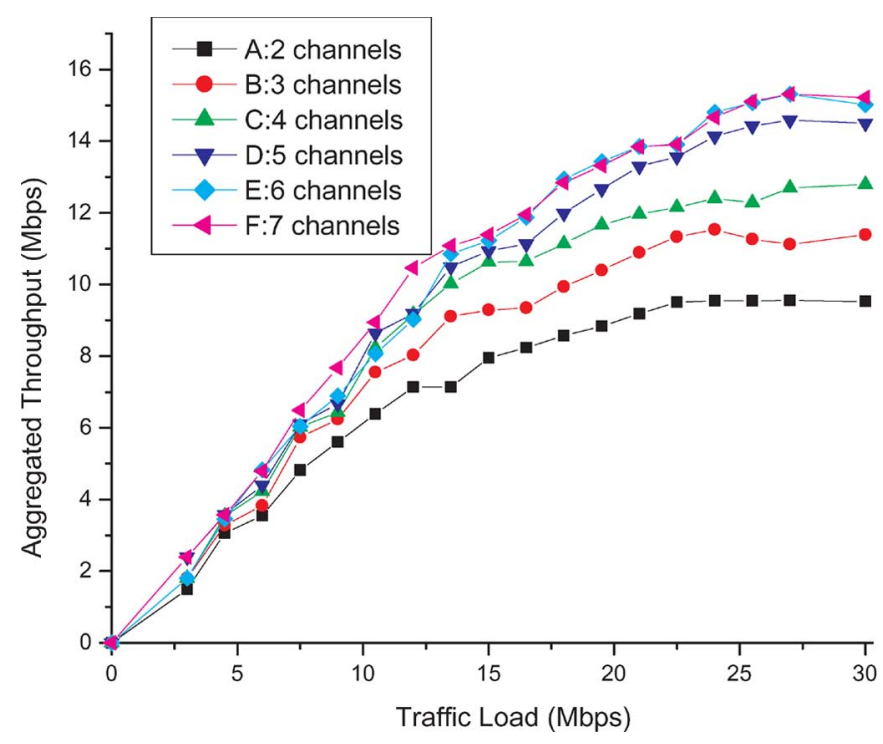

Fig. 14. Impact of increasing the number of available channels for random topology.

four available channels varies from 3 to 1.2 , depending on the topology. Similarly, for channel and routing adjustment, the normalized throughput varies from 2 to 1.1, and for power and routing adjustment, the normalized throughput varies from 1 to 1.4. The result suggests that joint adjustment is better than a separated channel or a power-related adjustment. Moreover, the improvement obtained with joint adjustment strongly depends on the underlying topology.

Fig. 14 shows the effects of varying the number of available channels on the network throughput. The number of orthogonal channels in the network varies between two and seven. The experimental setup for these simulations is the same (30 randomly placed nodes with five flows). We also vary the traffic load on each flow to evaluate the impact of traffic load on the utilization efficiency of the channels. The network throughput monotonically increases with the increase in traffic load when the number of available channels is kept constant because an interfering link group can be broken into smaller 


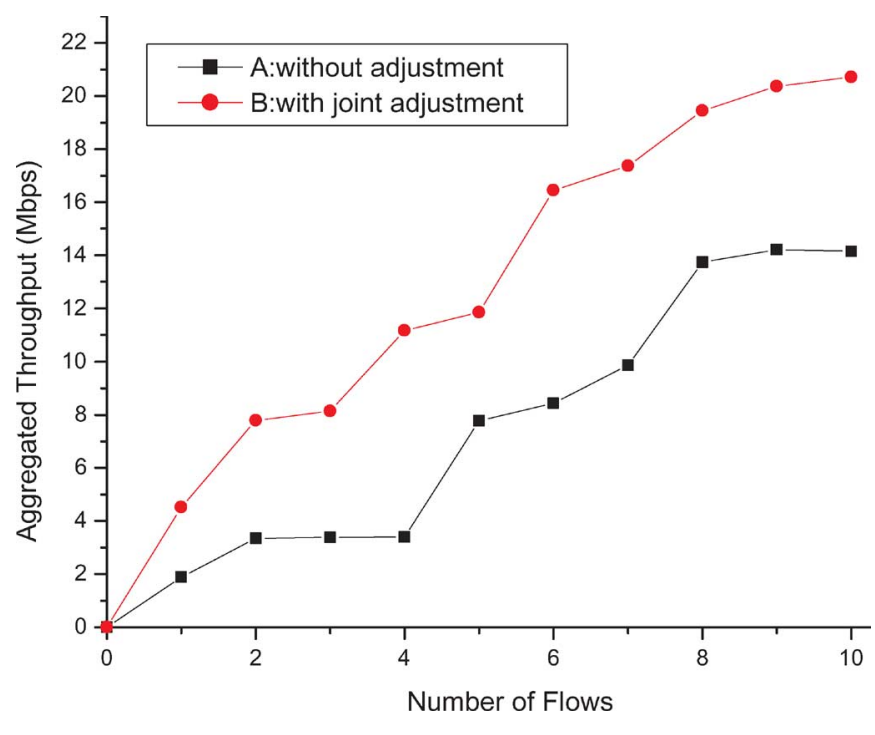

Fig. 15. Aggregated throughput with varying number of flows.

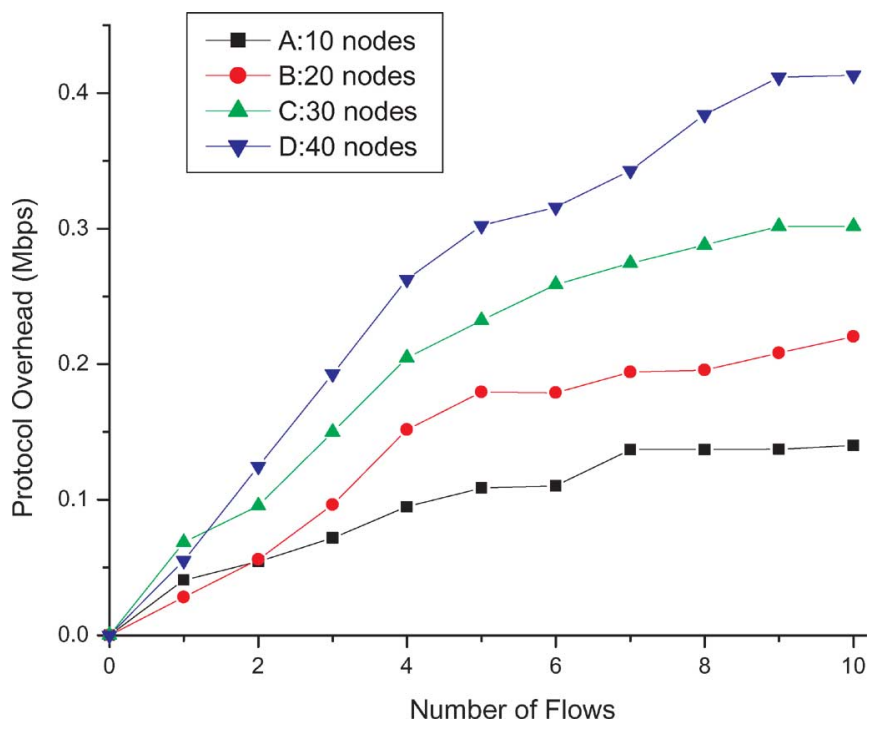

Fig. 16. Aggregated overhead with varying number of flows and nodes.

noninterfering link groups. When three channels are available, the network throughput saturates at about $9.5 \mathrm{Mb} / \mathrm{s}$. When the number of available channels is increased to six, the network throughput starts to saturate at $15 \mathrm{Mb} / \mathrm{s}$. After that, the increase in channels does not help to further improve the network throughput.

We also evaluate the impact of varying the number of flows in the network. Fig. 15 compares the throughput of joint adjustment with no adjustment. Ten flows are set up between randomly chosen node pairs. The traffic load on each flow is $4.5 \mathrm{Mb} / \mathrm{s}$. It is apparent that joint adjustment offers significantly better performance than network without adjustment, which is, on the average, two times the throughput of network without adjustment.

Fig. 16 plots the overhead of joint adjustment. The overhead includes the cost of sending link probe packet for measuring packet loss ratio, neighbor information exchange, and adjustment negotiation. The aggregated overhead monotonically increases with the number of nodes and flows. Nevertheless, the overhead per node remains small and consumes only a small fraction of the available channel capacity $(11 \mathrm{Mb} / \mathrm{s})$.

\section{RELATED WORK}

We are not aware of any other work that jointly considers topology control, which further consists of channel assignment, power control, as well as routing in multiradio multichannel wireless mesh networks, although there are quite a few works that are related to some relevant aspects.

For a related theoretical work, routing has been jointly considered with scheduling or power control in [16], [17], and [23]. Alicherry et al. [11] and Kodialam and Nandagopal [18] studied the channel assignment and routing problem to characterize the capacity region or to optimize the overall network throughput. However, all these theoretical works assume a perfect MAC without considering detailed IEEE 802.11 behavior.

Concerning power control, [19] proposed a localized algorithm to construct an energy-efficient topology. Reference [20] aimed to find a connectivity-preserving and interference minimum topology. There are a lot of other works that address power control and routing [21], [22]. However, most works were proposed in a single radio scenario without considering channel assignment.

Some recent works studied the problem of finding efficient routes to maximize throughput in a multiradio environment [7], [8]. Reference [7] utilized two radios to improve the performance of an ad hoc network and proposed a routing metric called the WCETT. Based on this work, [8] took channel switching overhead into account in the metric design. These studies consider only routing strategies for increasing the throughput.

For joint channel assignment and routing, [4], [5], [9], and [10] proposed various centralized or distributed algorithms for channel assignment and routing. However, these heuristic algorithms did nothing with transmission power control. Among them, [10] proposed a channel performance metric, called $C C M$, for joint channel assignment and routing. Our metric is distinct from $C C M$ in that the influence of spatial reusability is incorporated.

Therefore, all of these previous studies consider only a subset of the problem. None of them jointly considers the channel assignment, transmission power control, and routing for multiradio multichannel wireless mesh networks.

\section{CONCLUSION}

Observing the close interaction among channel assignment, power control, and routing, in this paper, we proposed a JTCR protocol for multichannel multiradio wireless mesh networks. By taking not only channel diversity but also spatial reusability into account, we improve the network performance through the joint adjustment of channel, transmission power, and route in a distributed way. A new metric ECATM is presented, which is utilized to evaluate the channel condition and quantify the performance gain of various adjustment candidates. The essential part of our protocol is to select a feasible adjustment candidate with the smallest metric value and then to coordinate 
the affected nodes through negotiation to realize the adjustment. Through an extensive simulation, we have demonstrated that network throughput can be significantly improved by using our proposed solution. It is part of our ongoing work to extend these results by further addressing the channel switching delay and the heterogeneity of radio interfaces. In addition, we plan to set up a testbed and perform real experimental testing to verify our proposed scheme.

\section{APPENDIX A \\ Connectivity Check Algorithm FOR CHANNEL AdJUSTMENT}

The neighbor nodes of node $i$ on channel $c$ have several properties (CommonRadioNumber, Active, TwoHopPath Exist). Among them, CommonRadioNumber $\in\{1, m\}$ means that there are one or more common radios with node $i$. Active $\in\{t, f\}$ represents whether the link that is connected with node $i$ is active or not. Similarly, TwoHopPathExist $\in$ $\{t, f\}$ denotes whether there is an alternative two-hop path between node $i$ and this neighbor. For example, $N_{i}(1, t / f, t)$ refers to a neighbor set that has one radio that is connected with node $i$. The link is active or inactive, and there is a two-hop path between node $i$ and these neighbors.

We summarize the channel adjustment check in the following procedure. It will return false if the connectivity cannot be kept or chain propagation appears; otherwise, it returns nodes set $C A N$ odeSet that should switch channel with $i$ and nodes set I ANodeSet that should switch their traffic flows to another interface.

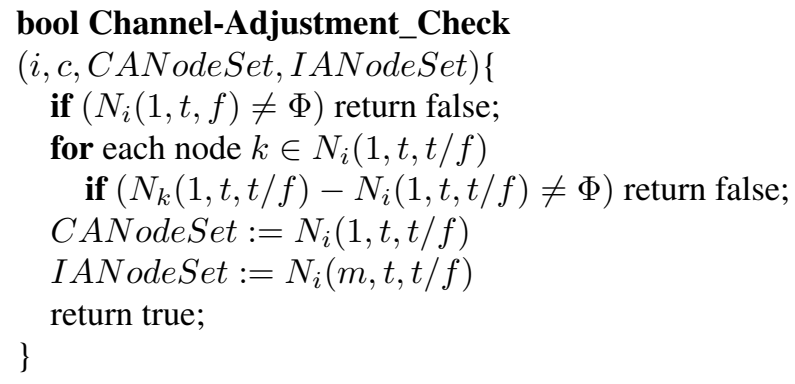

\section{APPENDIX B \\ Check Algorithm for Power Adjustment}

The neighbors of node $i$ on channel $c$ have several properties (PowerLevel, CommonRadioNumber, Active, TwoHop PathExist). Compared with the properties in channel adjustment check, PowerLevel $\in\left\{p_{l}, p_{h}\right\}$ is added to denote the neighbors that are reachable from $i$ under a lower or a higher power level. For example, $N_{i}\left(p_{h}, 1, t / f, t\right)$ refers to neighbors set that is reachable only through a higher power level and has one radio connected with node $i$. The links are active or inactive, and there are two-hop paths between node $i$ and these neighbors.

The following algorithm will return false if the connectivity cannot be kept or chain propagation happens; otherwise, it returns three set of nodes. PANodeSet is the neighbors set that should adjust power with $i, R A N$ odeSet is the neighbors set that should replace their original active links to the two-hop path, and I ANodeSet is the neighbors set that should switch the traffic flow to another interface.

bool Power-Adjustment_Check

( $i, P$ ANodeSet, RANodeSet, I ANodeSet) \{

if $\left(N_{i}\left(p_{h}, 1, t / f, f\right) \neq \Phi\right)$ return false;

// to avoid chain propagation

for each node $k \in N_{i}\left(p_{l}, 1 / m, t, t / f\right) \cup N_{i}\left(p_{h}, 1, t, t\right)$

if $\left(N_{k}\left(p_{l}, 1 / m, t, t / f\right) \cup N_{k}\left(p_{h}, 1, t, t\right)-\right.$

$\left.N_{i}\left(p_{l}, 1 / m, t, t / f\right) \cup N_{i}\left(p_{h}, 1, t, t\right) \neq \Phi\right)$

return false;

PANodeSet $:=N_{i}\left(p_{l}, 1 / m, t, t / f\right) \cup N_{i}\left(p_{h}, 1, t, t\right)$;

$R A N$ odeSet $:=N_{i}\left(p_{h}, 1, t, t\right)$;

IANodeSet $:=N_{i}\left(p_{h}, m, t, t / f\right)$;

for each node $k \in N_{i}\left(p_{h}, m, t / f, t / f\right) \cup N_{i}\left(p_{h}, 1, f, t\right)$

// potentially split more noninterfering link groups

if $\left(N_{k}\left(p_{l}, 1 / m, t, t / f\right) \cap(P A N o d e S e t)=\Phi\right)$

insert $N_{k}\left(p_{l}, 1 / m, t, t / f\right)$ into PANodeSet;

// chain propagation appears

else return false;

return true;

\}

\section{APPENDIX C \\ JTCR ALGORITHM}

void JTCR_ALGORITHM $(i$, LinkSet $)\{$

$/ /$ check the channel conditions

for each channel $c \in\left\{c_{1}, c_{2}, \ldots, c_{L}\right\}\{$ analyze links contention on channel $c$ in two- hop range; generate $G$ noninterfering groups on channel $c$; calculate $E C A T M$ on channel $c$ and $F_{g}^{c}$ for each group;

\}if (none of the channel node $i$ resides overloaded) return;

// find which channel is overloaded or least used find out the leastChan and overChan in terms of $F_{g}^{c}$,

// check the feasibility of channel adjustment

conFeasible $:=$ Channel-Adjustment_Check

( $i$, overChan, CANodeSet);

if (conFeasible) \{

caFeasible $:=$ Performance_Check () ;

\}// check the feasibility of power adjustment

conFeasible $:=$ Power-adjustment_Check

( $i$, PANodeSet, RANodeSet, I ANodeSet);

if (conFeasible) \{

for each node $k \in R A N$ odeSet \{

find a two-hop path to replace link between $i$ and $k$ \}paFeasible $:=$ Performance_Check () ;

\}if (caFeasible or paFeasible) \{ select adjustment candidate with minimal ECATM; begin negotiation;

\})

\section{REFERENCES}

[1] R. Bruno, M. Conti, and E. Gregori, "Mesh networks: Commodity multihop ad hoc networks," IEEE Commun. Mag., vol. 43, no. 3, pp. 123-131, Mar. 2005.

[2] IEEE 802.11 Working Group, Wireless LAN Medium Access Control (MAC) and Physical Layer (PHY) Specifications, 1997 
[3] IEEE 802.11a Working Group, Wireless LAN Medium Access Control (MAC) and Physical Layer (PHY) Specifications-Amendment 1: HighSpeed Physical Layer in the $5 \mathrm{GHz}$ Band, 1999.

[4] A. Raniwala, K. Gopalan, and T. Chiueh, "Centralized channel assignment and routing algorithms for multi-channel wireless mesh networks," ACM SIGMOBILE Mobile Comput. Commun. Rev., vol. 8, no. 2, pp. 50-65, Apr. 2004.

[5] J. Tang, G. Xue, and W. Zhang, "Interference aware topology control and QoS routing in multi-channel wireless mesh networks," in Proc. Mobihoc, 2005, pp. 68-77.

[6] M. Marina and S. Das, "A topology control approach for utilizing multiple channels in multi-radio wireless mesh networks," in Proc. Broadnets, 2005, vol. 1, pp. 381-390.

[7] R. Draves, J. Padhye, and B. Zill, "Routing in multi-radio, multi-hop wireless mesh networks," in Proc. Mobicom, 2004, pp. 114-128.

[8] P. Kyasanur and N. Vaidya, "Routing and link layer protocols for multichannel multi-interface ad hoc wireless networks," ACM SIGMOBILE Mobile Comput. Commun. Rev., vol. 10, no. 1, pp. 31-43, Jan. 2005.

[9] A. Raniwala and T. Chiueh, "Architecture and algorithms for an IEEE 802.11-based multi-channel wireless mesh network," in Proc. Infocom, 2005, vol. 3, pp. 2223-2234.

[10] H. Wu, F. Yang, K. Tan, J. Chen, Q. Zhang, and Z. Zhang, "Distributed channel assignment and routing in multi-radio multi-channel multi-hop wireless networks," IEEE J. Sel. Areas Commun.-Special Issue MultiHop Wireless Mesh Networks, vol. 24, no. 11, pp. 1972-1983, Nov. 2006.

[11] M. Alicherry, R. Bhatia, and L. Li, "Joint channel assignment and routing for throughput optimization in multi-radio wireless mesh networks," in Proc. Mobicom, 2005, pp. 58-72.

[12] D. De Couto, D. Aguayo, J. Bicket, and R. Morris, "High throughput path metric for multi-hop wireless routing," in Proc. Mobicom, 2003, pp. 134-146.

[13] V. Shah and S. Krishnamurthy, "Handling asymmetry in power heterogeneous ad hoc networks: A cross layer approach," in Proc. ICDCS, Jun. 2005, pp. 749-759.

[14] D. Johnson, D. Maltz, and J. Broch, DSR: The dynamic source routing protocol for multi-hop wireless ad hoc networks, 2004. IETF Manet Working group (Draft 10).

[15] W. Fang, N. Seddigh, and B. Nandy, A Time Sliding Window Three Colour Marker (TSWTCM), Jun. 2000. RFC 2859.

[16] R. L. Cruz and A. V. Santhanam, "Optimal routing, link scheduling and power control in multi-hop wireless networks," in Proc. Infocom, 2003, vol. 1, pp. 702-711.

[17] M. Kodialam and T. Nandagopal, "Characterizing achievable rates in multi-hop wireless networks: The joint routing and scheduling problem," in Proc. Mobicom, 2003, pp. 42-54.

[18] M. Kodialam and T. Nandagopal, "Characterizing the capacity region in multi-radio multi-channel wireless mesh networks," in Proc. Mobicom, 2005, pp. 73-87.

[19] W. Song, Y. Wang, X. Li, and O. Frieder, "Localized algorithms for energy efficient topology in wireless ad hoc networks," in Proc. Mobihoc, Dec. 2004, vol. 10, pp. 911-923. Issue 6.

[20] M. Burkhart, P. Rickenbach, R. Wattenhofer, and A. Zollinger, "Does topology control reduce interference," in Proc. Mobihoc, 2004, pp. 9-19.

[21] C. Yu, B. Lee, and H. Youn, "Energy efficient routing protocols for mobile ad hoc networks," Wirel. Commun. Mobile Comput., vol. 3, no. 8, pp. 959-973, Dec. 2003.

[22] P. Bergamo, A. Giovanardi et al., "Distributed power control for energy efficient routing in ad hoc networks," Wirel. Netw., vol. 10 no. 1, pp. 29-42, 2004.

[23] Y. Wu, P. Chou, Q. Zhang, K. Jain, W. Zhu, and S. Kung, "Network planning in wireless ad hoc networks: A cross-layer approach," IEEE J. Sel. Areas Commun., vol. 23, no. 1, pp. 136-150, Jan. 2005.

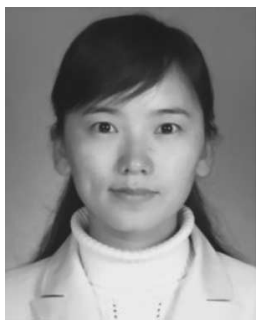

Lin Chen received the B.S. and M.S. degrees in computer science from Chongqing University of Posts and Telecommunications, Chongqing, China, in 1999 and 2002, respectively. She is currently working toward the Ph.D. degree with Shanghai Jiao Tong University, Shanghai, China.

Her research interests include resource management in wireless ad hoc networks and wireless sensor networks.

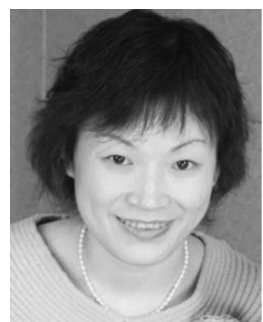

Qian Zhang (M'00-SM'04) received the B.S., M.S., and $\mathrm{Ph} . \mathrm{D}$. degrees from Wuhan University, Wuhan, China, in 1994, 1996, and 1999, respectively, all in computer science.

She was the Research Manager with the Wireless and Networking Group, Microsoft Research Asia, Beijing, China, in July 1999. She joined Hong Kong University of Science and Technology, Clear Water Bay, Hong Kong, in 2005, where she is an Associate Professor. She has published more than 150 refereed papers in leading international journals and key conferences in the areas of wireless/Internet multimedia networking, wireless communications and networking, and overlay networking. She also participated in many activities with the Internet Engineering Task Force Robust Header Compression working group for TCP/IP header compression. She is the holder of about 30 pending international patents. Her current research interests are in the areas of wireless communications, IP networking, multimedia, P2P overlay, and wireless security.

Dr. Zhang was the recipient of the TR 100 (MIT Technology Review) World's Top Young Innovator Award, the Best Asia Pacific (AP) Young Researcher Award elected by IEEE Communications Society in 2004, the Best Paper Award from the Multimedia Technical Committee of the IEEE Communication Society in 2005, the Best Paper Award for QShine 2006, and the Oversea Young Investigator Award from the National Natural Science Foundation of China in 2006. She is an Editorial Board Member of the IEEE TRANSACTIONS ON MULTimedia, the IEEE TRANSACTIONS ON WiRELESS COMMUNICATIONS, Elsevier Computer Communications, and Elsevier Computer Networks, as well as an Associate Editor for the IEEE TRANSACTIONS ON Vehicular TEChNOLOGY. She served as a Guest Editor for a special issue on wireless video of the IEEE Wireless Communication Magazine. She is now also serving as a Guest Editor for a special issue on Cross-Layer Optimized Wireless Multimedia Communications for the IEEE JOURNAL ON SELECTED AREAS In COMMUNICATIONS, Guest Editor for a special issue on Wireless Sensor Networking of the IEEE Wireless Communication Magazine, Guest Editor for a special issue on Wireless Multimedia Sensor Networks of Elsevier Computer Networks, and Guest Editor for a special issue on Wireless Mesh Networks in the ACM/Springer Journal of Mobile Networks and Applications.

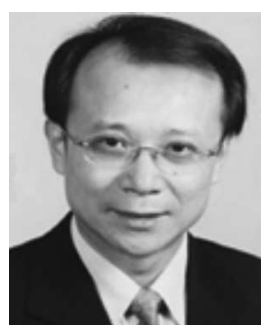

Minglu Li (M'05-A'05) received the B.S. degree from the University of Information Engineering, Zhenzhou, China, in 1985 and the Ph.D. degree in computer software from Shanghai Jiao Tong University, Shanghai, China, in 1996.

$\mathrm{He}$ is a Full Professor and Vice Chair of the Department of Computer Science and Engineering, Shanghai Jiao Tong University, where he is also the Director of the Grid Center. He has published over 100 papers in important academic journals and international conferences. His current major research interests include grid computing, wireless networks, peer-to-peer computing, and sensor networks.

$\mathrm{Mr}$. Li is an Associate Editor of the International Journal of Grid and Utility Computing and is a member of the editorial board of the International Journal of Web Services Research.

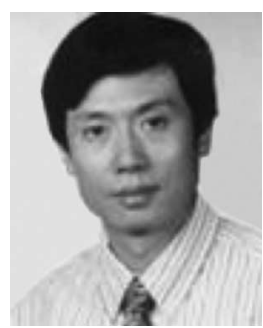

Weijia Jia received the B.Sc. and M.Sc. degrees from Center South University, Changsha, China, and the Ph.D. degree from the Polytechnic Faculty of Mons, Mons, Belgium, all in computer science.

$\mathrm{He}$ was a Research Fellow with German National Research Center for Information Science, St. Augustin, Germany, in 1993. He joined the Department of Computer Science, City University of Hong Kong, Kowloon, Hong Kong, in 1995 as an Associate Professor. His research interests include wireless communication and networks, distributed systems, multicast, and anycast QoS routing protocols for the Internet. In these fields, he has published more than 200 papers and books/chapters in international journals and conference proceedings. $\mathrm{He}$ has been the Principal-Investigator of more than 20 research projects that are supported by RGC Research Grants, Hong Kong, and CityU Strategic Research Grants. Currently, he is in charge of a HK \$10 million ITF project supported by the Hong Kong Government for the development of Next-Generation Ubiquitous Communication Platforms.

Mr. Jia served as a PC Cochair and PC Member for various IEEE international conferences. 